\title{
Approaches For A Destructive Measurement Method Of Subsurface Damages
}

\author{
Michael Seiler ${ }^{1,}$, Lukas Tianis ${ }^{1}$, Jens Bliedtner $^{1}$, Mario Berlinger ${ }^{2}$ Steffen Gürtler ${ }^{2}$ \\ ${ }^{1}$ Ernst-Abbe-Hochschule Jens, Department SciTec, Carl-Zeiss-Promenade 2, 07745 Jena, Germany \\ ${ }^{2}$ Bühler Alzenau GmbH, Föpplstraße 9, 04347 Leipzig, Germany
}

\begin{abstract}
In optical manufacturing, the depth of subsurface damages (SSD) depicts an important quality aspect of optical elements. Nowadays, the investigation methods of the damage zone are mostly destructive and allow a sampling of the substrates only. This leads to a lack of statistical reliability. In this paper, one aim is to develop an easy and cheap procedure to evaluate the probes. Therefore, a spherical polishing and chemical etching is introduced. By means of imaging processing software the acquired data is analysed, which eliminates the operator influence und increases the reproducibility. Applying binarizing and shape detection algorithms the SSD structures is detected and the number of pixels per structure is calculated. The percentage of SSD over the depth fit with an exponential curve displays a maximum depth as a single value. The development of a method to choose the best threshold value represents an approach to quantify the uncertainty of the resulting SSD-depth.
\end{abstract}

\section{Introduction}

The conventional optical manufacturing processes, such as grinding and lapping, leave a damaged layer, which includes micro cracks. These subsurface damages (SSD) are one of the important quality characteristics of optical elements. One major interest is the evaluation of the depth of the damaged zone which determines the material removal for the following polishing pro-cess and, thereby, the time and cost. Nowadays, most of the used techniques to investigate SSD are destructive and only allow sampling to test the processed substrates [1]. This leads to a lack of statistical reliability of the measurement process.

\section{Experimental results}

The development of the new procedure shown in Fig. 1 for the preparation of samples of N-BK7, SF6 and HPFS7980 by spherical polishing and chemical etching was the first aim. The elimination of operator influences and increase of reproducibility of the evaluation process was another. To do so, a self-developed image processing software analyses the microscope photographs of the prepared surface taken on random positions of the etched surface. Binarizing and shape-detection algorithms are the key functions to detect SSD structures.

Fig. 2 displays some typical SSD-structures after etching. The SSD are marked in red. In order to get the percentage of SSD, the number of pixels is calculated.
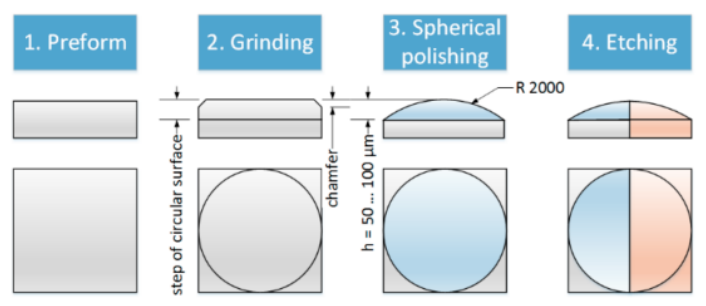

Fig. 1. Spherical Polishing process.

The spherical shape of the specimen is measured by a contact stylus profilometer. Due to the single profiles the depth of removal at any distance from the center of the specimen is provided.

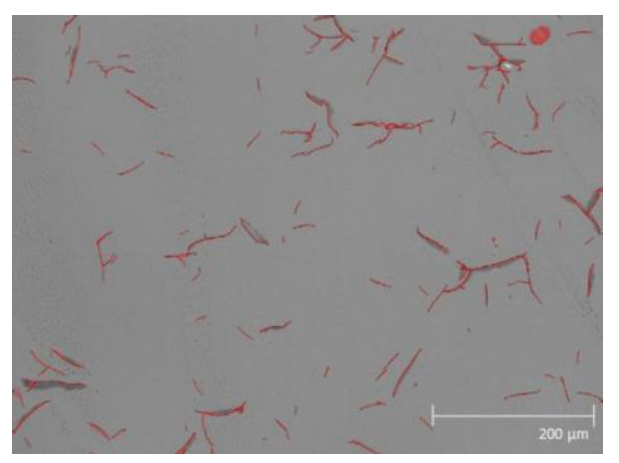

Fig. 2. SSD evaluation (marked red) with digital microscope.

\footnotetext{
*Corresponding author: michael.seiler@eah-jena.de
} 
To investigate the SSD itself, laser-scanning microscopy (LSM) provides images of the topography. Images of the unetched surface identify peaks above the surface level.

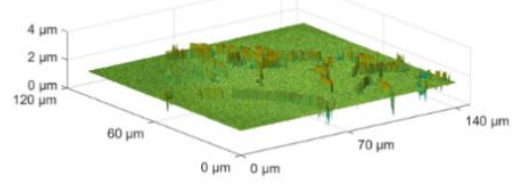

Fig. 3. LSM mapping of an unetched surface.

After wet etching, these structures increase in lateral dimension. At the contour map these structures display as valleys. It is presumed that the unetched structures create reflections of the laser, which lead to inappropriate measurement result. After etching, the structures are wided and the reflection are eliminated. Consequently, the examined structures are subsurface-damages. To further examine the SSD, a digital light microscope acquires the images, because there is no need to gain data of the height of the surface. Light microscopy can detect SSD with sufficient precision. An examination of the different possibilities to recognize SSD on etched and non-etched specimen by taking images of the transition zone between the two parts of the specimen is performed.

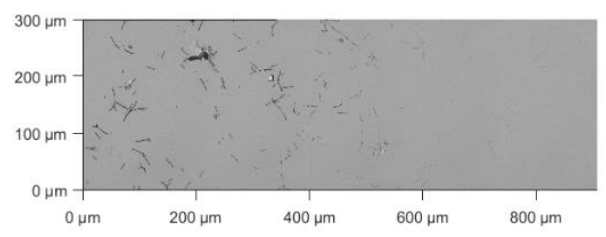

Fig. 4. Comparison between unetched and etched region on a N-BK7 surface.

Fig. 4 shows the transition zone. SSD on the etched part (left) occur as dark structures whereas on the unetched part (right) no SSD can be identified. Summarized, an etching of the specimen is indispensable the evaluate SSD.

By linking the surface profile with the positions of the microscope photographs and the calculated percentage of SSD, a plot of SSD-density over the depth of removal is created. As shown in Fig. 5, the data can be described by an exponential fit shown in Eq. (1). \%SSD is the percentage of SSD $d_{r}$, the depth of removal and $a, b$ the coefficients of the fit.

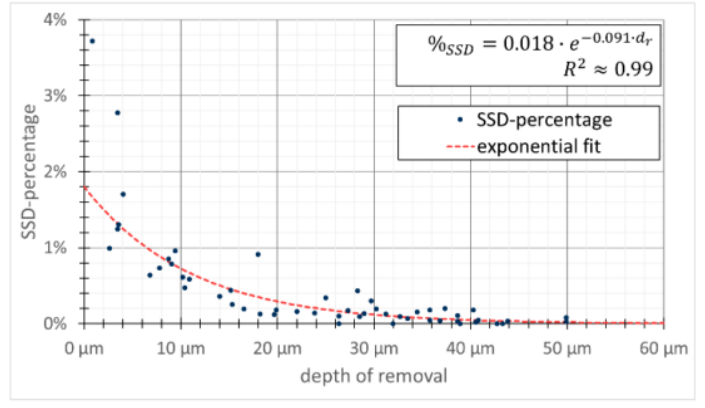

Fig. 5. Exponential Fitting of the SSD-percentage over depth of removal.

$$
\%_{S S D}=a \cdot e^{b \cdot d_{r}}
$$

After executing the test for outliers and eliminating these data, the coefficient of determination is in general higher than 0.95 . Threshold values applied on the fitted curve describe the maximum depth of SSD as a single value. For that, Eq. (1) is resolved to $x$ and the threshold value is used for $\mathrm{f}(x)$ in Eq. (2), the fit.

$$
d_{r}=\ln \frac{\% S S D}{a} \cdot \frac{1}{b}
$$

The threshold value has an immediate influence on the statistical reliability and, therefore, needs to be chosen prudently.

\section{Summary and Outlook}

A Comparison of the different glasses and the examination of the whole measurement process will describe its suitability. The development of a method to choose the best threshold value represents an approach to quantify the uncertainty of the resulting SSD-depth. Additionally, an improvement of the software should be sought to detect SSD with high precision.

Furthermore, additional materials will be tested. The detection of the SSD shape can be enhanced with object detection method and further machine learning.

\section{Acknowledgements}

Work is performed under the auspices of the German Federal Ministry for Economic Affairs and Energy under funding reference ZF4039605PR6.

The authors would like to thank Dietmar Gräfe and Sebastian Henkel for processing the samples.

\section{References}

1. Y. Li, J. Wang, Q. Xu and Y. Guo, Journal of the European Optical Society - Rapid Publications, (2011) 